\title{
Targeting PKCı-PAK1 in EGFR-mutation positive non-small cell lung cancer
}

\author{
Masaoki Ito ${ }^{1}$, Carles Codony-Servat ${ }^{1}$, Niki Karachaliou ${ }^{2}$, Rafael Rosell $^{3}$ \\ ${ }^{1}$ Coyote Research Group, Pangaea Oncology, Laboratory of Molecular Biology, Quiron-Dexeus, University Institute, Barcelona, Spain; ${ }^{2}$ Institute of \\ Oncology Rosell (IOR), University Hospital Sagrat Cor, QuironSalud Group, Barcelona, Spain; ${ }^{3}$ Catalan Institute of Oncology, Hospital Germans \\ Trias i Pujol, Badalona, Spain \\ Contributions: (I) Conception and design: All authors; (II) Administrative support: All authors; (III) Provision of study materials: All authors; (IV) \\ Collection and assembly of data: All authors; (V) Data analysis and interpretation: All authors; (VI) Manuscript writing: All authors; (VII) Final \\ approval of manuscript: All authors. \\ Correspondence to: Rafael Rosell. Institut d'Investigació en Ciències de la Salut Germans Trias i Pujol Campus Can Ruti (Edifici Muntanya), Ctra. de \\ Can Ruti, Cami de les Escoles s/n, 08916 Badalona, Barcelona, Spain. Email: rrosell@iconcologia.net.
}

Background: Epidermal growth factor receptor (EGFR) tyrosine kinase inhibitors (TKIs) induce significant responses in EGFR-mutation positive non-small cell lung cancer (NSCLC). However, universal progression is observed.

Methods: The effect of the anti-rheumatoid agent, auranofin, a selective inhibitor of oncogenic protein kinase $\mathrm{C}$ iota $(\mathrm{PKCl})$ signaling and IPA-3, a non-ATP competitive p21-activated kinase 1 (PAK1) inhibitor in treatment-naïve and EGFR TKI-resistant EGFR-mutation positive NSCLC cell lines was investigated. PC9 and HCC827 cells were used. The four EGFR-TKI resistant cell lines were established from PC9. Cell viability assays, drug combination studies, and western blotting were performed. The combination index, and RTK or non-RTK expression were performed.

Results: The combination of IPA-3 and auranofin was highly synergistic in all 6 cell lines (combination indexes ranged from 0.37-0.62). The activities on EGFR, CDCP1, AXL, MET, and downstream effector pathways, including PAK1, PKC1, ERK, AKT, STAT3, Src, and YAP1 were abrogated.

Conclusions: The combination of auranofin with IPA-3 could be a potential therapy for EGFR-mutation positive NSCLC resistant to EGFR TKIs. Auranofin with IPA-3 could become a therapeutic solution for EGFR-mutation positive NSCLC patients resistant to EGFR TKIs.

Keywords: Auranofin; epidermal growth factor receptor tyrosine kinase inhibitors (EGFR-TKIs); IPA-3; nonsmall cell lung cancer (NSCLC); resistance

Submitted Jul 02, 2019. Accepted for publication Aug 08, 2019.

doi: $10.21037 /$ tlcr.2019.08.25

View this article at: http://dx.doi.org/10.21037/tlcr.2019.08.25

\section{Introduction}

Epidermal growth factor receptor (EGFR) tyrosine kinase inhibitors (TKIs) induce significant responses and reasonably prolonged progression-free survival of 10 to 16 months in EGFR-mutation positive non-small cell lung cancer (NSCLC). However, universal progression is observed $(1,2)$. Overexpression of receptor tyrosine kinases (RTKs) and downstream components have been reported (1). Gefitinib and osimertinib (first- and thirdgeneration EGFR TKIs, respectively) suppress EGFR, extracellular signal-regulated protein kinase 1/2 (ERK1/2), and phosphatidylinositol 3-kinase $(\mathrm{PI} 3 \mathrm{~K}) /$ protein kinase $\mathrm{B}$ (PKB, also called AKT) phosphorylation, but increase signal transducer and activator of transcription 3 (STAT3), paxillin and yes-associated protein 1 (YAP1) phosphorylation in PC9 cells (exon 19 deletion). Co-expression of AXL, CUBdomain-containing protein 1 (CDCP1), and MET is 
Table 1 Characteristics of cell lines used in the study

\begin{tabular}{llcc}
\hline Cell lines & EGFR mutation & Clinical profile & Molecular profile \\
\hline PC9-GR3 & Exon 19 deletion & Gefitinib-resistant & MET overexpression (9) \\
PC9-GR4 & Exon 19 deletion & Gefitinib-resistant & EGFR T790M (9) \\
PC9-OR2 & Exon 19 deletion & Osimertinib-resistant & - \\
PC9-OR4 & Exon 19 deletion & Osimertinib-resistant & Elevation of AXL (data not shown) \\
\hline
\end{tabular}

evident in PC9 cells, which is abolished when YAP1, or the Src-family kinases, YES or LYN, are knocked down. The combination of gefitinib or osimertinib with a pan-kinase inhibitor (repotrectinib) that blocks Src, focal adhesion kinase (FAK), and the janus kinase 2 (JAK2), downregulates the RTKs and their downstream effectors, and causes tumor growth inhibition (1). Currently, resistance to osimertinib poses an unsolved problem in EGFR-mutation positive NSCLC. Auranofin is an oral, U.S. Food and Drug Administration-approved, lipophilic gold-containing antirheumatoid compound (3). Auranofin is a selective inhibitor of the oncogenic protein kinase $\mathrm{C}$ iota (PKC iota) signaling (4-6). IPA-3 is a non-ATP competitive p21-activated kinase 1 (PAK1) inhibitor (7). PAK1 has been related with resistance to gefitinib, through a continuous activation of the PI3K/AKT/[CCAAT/enhancer binding protein beta $(\mathrm{C} /$ EBP- $\beta$ )]/miR-145 cascade (8). We investigated the effect of auranofin plus IPA-3 in treatment-naïve and EGFR TKIresistant EGFR-mutation positive NSCLC cell lines.

\section{Methods}

\section{Cell lines and reagents}

The human lung adenocarcinoma PC9 cell line, harboring EGFR exon 19 deletion was provided by F. HoffmannLa Roche Ltd. (Basel, Switzerland). EGFR exon 19 deletion positive HCC827 cells were purchased from the American Type Culture Collection (ATCC) (1). The two gefitinib-resistant (PC9-GR3 and PC9-GR4) cell lines, that we used in the present work, have been established from the PC9 cell line as previously described (9). The two osimertinib-resistant (PC9-OR2 and PC9-OR4) cell lines were generated by treating PC9 cells, with increasing concentrations of osimertinib. Sequencing analyses revealed that both cell lines retained the EGFR exon 19 deletion (Table 1). The half-maximal inhibitory concentration (IC50) for osimertinib of parental PC9 cells was in the nanomolar range compared to $3.1-3.7 \mu M$ in the resistant cell lines. Cell lines were cultured with RPMI (Roswell Park Memorial Institute medium) supplemented with $10 \%$ fetal bovine serum (FBS; Gibco), and $50 \mu \mathrm{g} / \mathrm{mL}$ penicillin/streptomycin/glutamine (Gibco) in a $5 \% \mathrm{CO}_{2}$ $37{ }^{\circ} \mathrm{C}$ cell culture incubator and routinely evaluated for mycoplasma contamination. The PAK1 inhibitor (IPA-3) was purchased from Selleck Chemicals (Houston, TX, U.S) and the PKC iota inhibitor (auranofin) was purchased from Sigma-Aldrich (St. Louis, MO, U.S). The total PKC iota, and the phospho-specific PKC iota/lamda (Thr555/Thr563) antibodies were purchased from Abcam (Cambridge, MA, US).

The $\beta$-actin antibody was purchased from Sigma Aldrich (St Louis, MO, US). The rest of phospho-specific and total antibodies used in this study were purchased from Cell Signaling Technology (Beverly, MA, US). The secondary antibodies Amersham ECL-anti-rabbit IgG horseradish peroxidase-linked species-specific whole antibody, and Amersham ECL-anti-mouse IgG peroxidase-linked whole antibody were purchased from GE Healthcare UK limited (Buckinghamshire, UK).

\section{Cell viability and growth assays}

Cells were cultured in 96-well plates. The number of cells for the viability assay was determined based on increasing viabilities of each cell line. Cells were seeded at the following densities $1 \times 10^{3}, 2 \times 10^{3}, 3 \times 10^{3}, 4 \times 10^{3}$, and $5 \times 10^{3}$, and incubated for $24 \mathrm{~h}$, as previously described $(1,2)$. After $72 \mathrm{~h}$ of incubation, MTT [3-(4,5-dimethylthiazol-2-yl)2,5-diphenyltetrazolium bromide] reagent (Sigma-Aldrich, St. Louis, MO, US) was added to the medium for $90 \mathrm{~min}$ at $37{ }^{\circ} \mathrm{C}$. Then, the culture medium was removed, and formazan crystals were reabsorbed in $100 \mu \mathrm{L}$ of dimethyl sulfoxide (DMSO), and quantified at $492 \mathrm{~nm}$ absorbance using a microplate reader. Cell viability was determined by measuring the absorbance at $492 \mathrm{~nm}$ using a Varioskan Flash 96-well microplate reader (Thermo Fisher Scientific, Massachusetts, US). The IC50 of each compound was 
Table 2 Half-maximal inhibitory concentration (IC50) of auranofin and IPA-3 in the cell lines used in the study

\begin{tabular}{lcc}
\hline Cell lines & Auranofin $(\mu \mathrm{M})$ & IPA-3 $(\mu \mathrm{M})$ \\
\hline PC9 & 3.25 & 22.5 \\
HCC827 & 2.5 & 30.0 \\
PC9-GR3 & 2.0 & 30.0 \\
PC9-GR4 & 2.5 & 30.0 \\
PC9-OR2 & 3.25 & 30.0 \\
PC9-OR4 & 3.25 & 30.0 \\
\hline
\end{tabular}

determined using GraphPad prism7 (GraphPad Soft Ware, San Diego, CA, US). For the interaction of drugs, cells were cultured with medium including 10 different concentrations of each agent (from agent-free to 3 times of the IC50). Data of combined drug effects were calculated by the Chou and Talalay method $(10,11)$. Combination indexes (CI) values $<0.6, \geq 0.6$ but $<0.8, \geq 0.8$ but $<0.9, \geq 0.9$ but $<1.1$, and $\geq 1.1$ indicate strong synergism, moderate synergism, slight synergism, additive interaction, and antagonism, respectively.

\section{Western blotting}

For immunoblotting experiments, cells were washed with cold phosphate-buffered saline (PBS) and re-suspended in ice-cold radioimmunoprecipitation assay (RIPA) buffer $(50 \mathrm{mM}$ Tris- hydrochloric acid in $\mathrm{pH} 7.4,1 \%$ Nonidet P-40, 0.5\% sodium deoxycholate, $0.1 \%$ sodium dodecyl sulfate (SDS), $150 \mathrm{mM}$ sodium chloride, $1 \mathrm{mM}$ ethylenediaminetetraacetic acid, $1 \mathrm{mM}$ sodium vanadate and $50 \mathrm{mM}$ sodium fluoride) containing protease inhibitor mixture (Roche Applied Science, Penzberg, Germany). Then cells were lysed by sonication and centrifugation at $14,000 \mathrm{rpm}$ (revolutions per minute of rotor) for $15 \mathrm{~min}$ at $4{ }^{\circ} \mathrm{C}$, and the resulting supernatant was collected as the total cell lysate. The lysates containing $30 \mu \mathrm{g}$ proteins were electrophoresed on 10\% SDS-polyacrylamide gel electrophoresis (Life Technologies, Carlsbad, CA, U.S.) and transferred to polyvinylidene difluoride membranes (Bio-Rad laboratories Inc., Hercules, CA, US). Odyssey blocking buffer (Li-Cor Biosciences, Lincoln, NE, US) was used to block the membranes. All target proteins were immunoblotted with appropriate primary and either IRDye-conjugated or horseradish peroxidase (HPR)conjugated secondary antibodies. Fluorescent bands
(IRDye-conjugated) were detected with Odyssey CLx Imager (Li-Cor Biosciences, Lincoln, NE, US), whereas chemiluminescent (HRP-conjugated) were detected in a ChemiDoc MP Imaging System (Bio-Rad laboratories Inc.). $\beta$-actin was used as an internal control to confirm equal gel loading. Primary and secondary antibodies used in the study was shown in Table S1.

\section{Ethical approval}

This study has not been reviewed by ethics committee because any samples from patients or animals were not used. This study was performed using cell lines.

\section{Results}

Single drug treatment with auranofin or IPA-3 of EGFRmutation positive cell lines

We first determined the inhibitory effects of auranofin and IPA-3 on the growth of our panel of EGFR-mutation positive cell lines. Of these cell lines, HCC827 and PC9 are treatment naïve, PC9-GR3 and PC9-GR4 are gefitinibresistant (9), and PC9-OR2 and PC9-OR4 are osimertinibresistant. As shown in Table 1, diverse mechanisms of resistance were observed in the four EGFR TKI-resistant cell lines. Specifically, the PC9-GR3 and the PC9-OR4 cell lines have MET (9) and AXL overexpression, respectively, compared to the parental PC9 cell line. AXL has been described as a mechanism of resistance to EGFR TKIs (12). PC9-GR4 cells have the acquired T790M resistant mutation (9). Auranofin and IPA-3 were tested in a doseresponse manner resulting in IC50 values in the range of $\mu \mathrm{M}$ (Table 2), demonstrating the lack of potential of these compounds alone for the treatment of our EGFR-mutation positive cell lines.

\section{Combination treatment with auranofin and IPA-3}

We then examined the combination of IPA-3 with auranofin in our EGFR-mutation positive cell lines. As shown in Figure $1 A$ (left panels) the combination augmented the effect of each drug alone on cell viability in a synergistic manner, in the two EGFR TKI naïve cell lines. We could detect a pronounced synergism between auranofin and IPA-3 with CIs of 0.55 and 0.37 in PC9 and HCC827 cells, respectively (Figure 1B). Surprisingly, the combination of auranofin and IPA-3 decreased proliferation more than either drug alone 

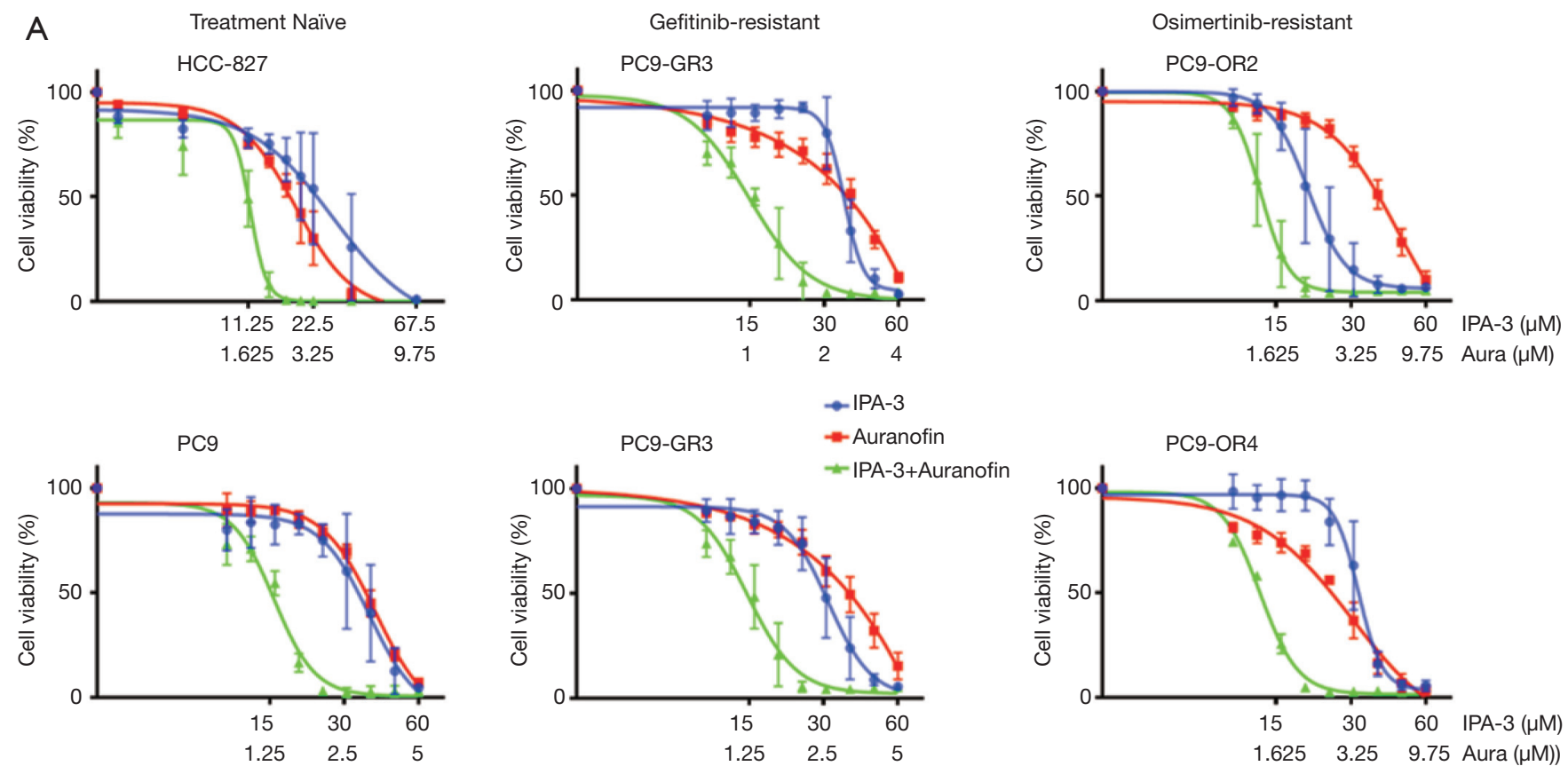

B Synergism Antagonism

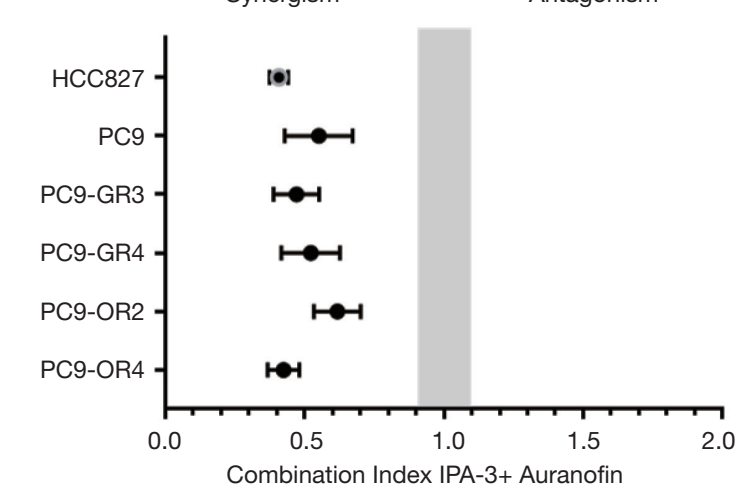

Figure 1 Sensitivity of EGFR-mutation positive cell lines to the combination of IPA-3 plus auranofin. (A) Dose response curves of the IPA-3 plus auranofin combination. Dose response curves of the combination of IPA-3 plus auranofin for the growth inhibition of two EGFR TKInaïve (HCC827, PC9) (left panels), two gefitinib resistant (PC9-GR3, PC9-GR4) (middle panels) and two osimertinib-resistant cell lines (PC9-OR2, PC9-OR4) (right panels), treated with varying concentrations of the two compounds for 72 hours. (B) Combination indexes obtained by the Chou-Talalay method combining auranofin plus IPA-3. The cell lines treated are indicated in the left side (y-axis) and the combination index is indicated in the $\mathrm{x}$-axis. The combination of IPA-3 plus auranofin was highly synergistic for all cell lines tested. The results presented in A and B are the average of at least three independent experiments and the error bars represent the standard deviation.

in the two gefitinib-resistant EGFR-mutation positive cell lines (Figure 1A, middle panels). Specifically, the CI was 0.47 for PC9-GR3 cells with MET overexpression, and 0.52 for PC9-GR4 with acquired EGFR T790M resistant mutation (Figure $1 B$ ). Similar results we obtained with the two osimertinib resistant EGFR-mutation positive cell lines (Figure 1A, right panels). A strong (CI, 0.42) synergism of auranofin and IPA-3 was observed in the PC9-OR4 cell line which overexpresses AXL (Figure 1B). In PC9-OR4 cells a moderate synergistic effect was obtained with a CI of 0.62 (Figure 1B). These data show that a combination without an EGFR inhibitor, synergistically decreases cellular proliferation in EGFR-mutation positive cells, even when diversity on mechanisms of resistance to EGFR inhibitors is present. 


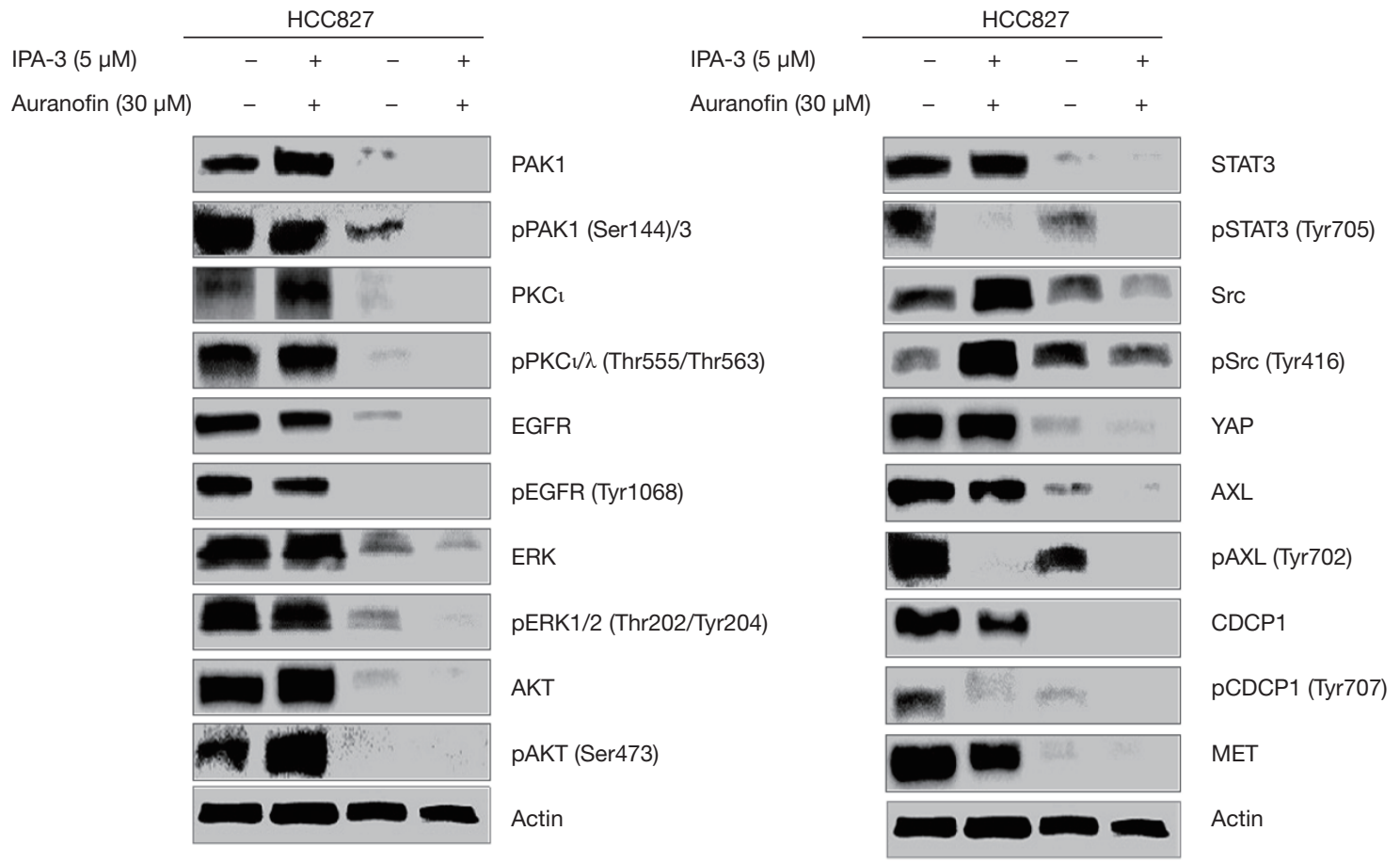

Figure 2 Effects of IPA-3 plus auranofin in an EGFR-mutation-positive NSCLC cell line. Extracts from the HCC827 cell line treated with IPA-3 $(5 \mu \mathrm{M})$, auranofin $(30 \mu \mathrm{M})$, or the double combination for 6 hours. A combination of IPA-3 plus auranofin abrogated EGFR, CDCP1, AXL, MET, and downstream effector pathways, including PAK1, PKC1, ERK, AKT, STAT3, Src, and YAP1. $\beta$-actin was used as an internal control to confirm equal gel loading. The double combination ablated the expression of YAP1, MET and Src. Similar results were obtained in three independent experiments.

\section{Correlation between signaling nodes and cellular responses to auranofin plus IPA-3 combination}

We then try to determine by western blotting which signaling nodes are abrogated with the combination of auranofin plus IPA-3 (Figure 2). For this, we selected the HCC827 cell line, in which the combination of the two drugs distinguished from the rest of the cell lines, with the lowest CI of 0.37. Extracts from the HCC827 cell line treated with IPA-3 $(5 \mu \mathrm{M})$, auranofin $(30 \mu \mathrm{M})$, or the double combination for 6 hours were used for immunoblotting. As expected, IPA-3 fully abolished total and phosphorylated PAK1 at serine 144/141 and auranofin completely abrogated total PKC iota and PKC iota and lambda phosphorylation at threonine 555 and 563, respectively. Auranofin significantly decreased the level of total and phosphorylated PAK1, in comparison with the untreated cells (Figure 2). We have previously reported AXL and CDCP1 activation in treatment-naïve EGFR mutation positive cell lines, that was abrogated by either silencing YAP1, Src, YES, and LYN (Src associated proteins), or by treating the cells with the Src/focal adhesion kinase (FAK)/JAK2 inhibitor (TPX0005, also known as repotrectinib). The combination of osimertinib and TPX-0005 was highly synergistic in vitro and in vivo (1). In the present study, IPA-3 alone abrogated STAT3 phosphorylation at tyrosine 705, as well as AXL and CDCP1 phosphorylation. We observed a striking activity of auranofin alone on most of the signaling modes explored in our study, including activated EGFR. The combination of IPA-3 plus auranofin abrogated EGFR, CDCP1, AXL, and downstream effector pathways, including ERK, AKT, STAT3, and Src. The double combination ablated the expression of YAP1 and MET (Figure 2). These results reinforce the relevance of co-targeting PAK1 and PKC iota in EGFR-mutation positive cell lines.

\section{Discussion}

PKC1-epithelial cell transforming 2 (Ect2)-Ras-related C3 botulinum toxin substrate 1 (RAC1) pathway is required for lung tumorigenesis (5). It has been shown that recalcitrant 


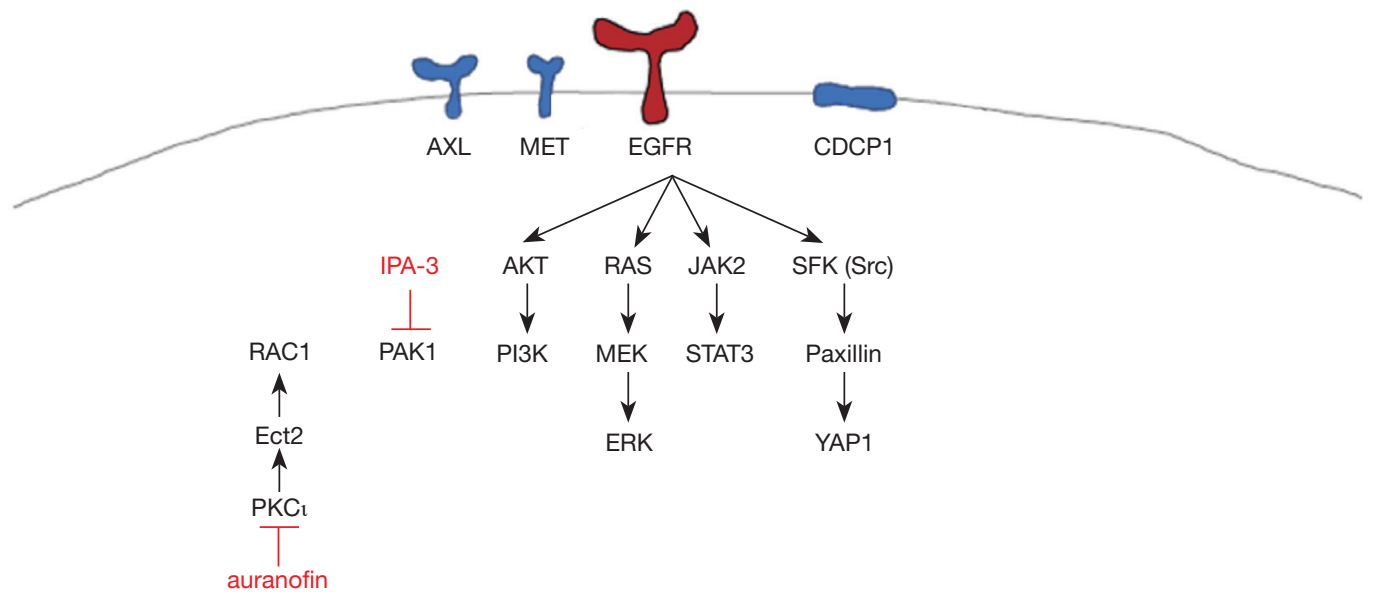

Figure 3 Our model. PAK1 activates the PI3K/AKT signaling pathway and PKC1 activates PAK1 through Ect2 and RAC1. Auranofin with IPA-3 (liposomal formulation), or other PAK inhibitors, could become a therapeutic solution for EGFR-mutation positive NSCLC patients resistant to EGFR TKIs.

activation of PI3K/AKT via feedback loop of PAK/ $\mathrm{PI} 3 \mathrm{~K} / \mathrm{AKT} / \mathrm{C} / \mathrm{EBP}-\beta / \mathrm{miR}-145$ can be solved with the combination of gefitinib plus IPA-3 (7). The combination inhibited tumor growth in PC9 and PC9- gefitinib-resistant xenografts (7). The combination of auranofin with ibrutinib, an inhibitor of Burton's tyrosine kinase, inhibits mutant EGFR by reversibly binding with Cys797. Auranofin plus ibrutinib inhibit the MEK/ERK, AKT/mTOR and STAT3 pathways (6).

In this study, we found that the combination of auranofin with IPA-3 could be a potential therapy for EGFR-mutation positive NSCLC treatment-naïve or resistant to first- or third-generation EGFR TKIs. The combination decreased cell viability in a synergistic manner in EGFR-mutation positive cells with diverse mechanisms of resistance to EGFR TKIs, including MET or AXL overexpression, or the acquisition of the secondary T790M mutation (Figure 1). Our model indicates that EGFR activation by tyrosine kinase domain mutations and the co-expression of RTKs and non-RTKs, including AXL, MET and CDCP1, sustain tumor cell proliferation and survival through the activation of downstream signaling pathways, like ERK/MAPK, the PI3K/AKT, the JAK2/STAT3 and the Src-YAP1 pathways. A combination that does not include an EGFR inhibitor was able to abolish all the upstream and downstream signaling nodes (Figures 2,3).

IPA-3 is a proof-of-concept compound that selectively targets PAK1 but represents only a laboratory tool. Other PAK1 inhibitors are in development (13), but blockade of PAK1 alone will be insufficient. On the other hand, auranofin is an anti-rheumatic drug used in clinic, and apart from targeting PKC iota has other anti-tumor properties (14). It is currently in clinical trials for different type of tumors, including lung cancer (ClinicalTrials. gov Identifier: NCT01737502). Auranofin with IPA-3 merits further preclinical evaluation and could become a therapeutic solution for EGFR-mutation positive NSCLC patients resistant to EGFR TKIs.

\section{Conclusions}

The combination of auranofin with IPA-3 could be a potential therapy for EGFR-mutation positive NSCLC resistant to EGFR TKIs. Auranofin with IPA-3 could become a therapeutic solution for EGFR-mutation positive NSCLC patients resistant to EGFR TKIs.

\section{Acknowledgments}

We would like to acknowledge the participation of Dr. Xiaoyan Sun for her contribution in screening the PAK1 inhibitors (Affiliated Hospital of Integrated Traditional Chinese and Western Medicine, Laboratory of Cellular and Molecular Biology, Nanjing, Jiangsu 210028, China).

Funding: Work in Dr Rosell's laboratory is partially supported by a grant from La Caixa Foundation and a Marie Skłodowska-Curie Innovative Training Networks European Grant (ELBA No 765492). Instituto de Salud 
Carlos III grant (RESPONSE, PIE16/00011) and a Spanish Association against Cancer (AECC) grant (PROYE18012ROSE). The work of Dr. Masaoki Ito in Pangaea Oncology is supported by an International Association for the Study of Lung Cancer (IASLC) Lung Cancer Fellowship Award 2017-2018.

\section{Footnote}

Conflicts of Interest: The authors have no conflicts of interest to declare.

Ethical Statement: The authors are accountable for all aspects of the work in ensuring that questions related to the accuracy or integrity of any part of the work are appropriately investigated and resolved.

\section{References}

1. Karachaliou N, Chaib I, Cardona AF, et al. Common co-activation of AXL and CDCP1 in EGFR-mutationpositive non-small cell lung cancer associated with poor prognosis. EBioMedicine 2018;29:112-27.

2. Chaib I, Karachaliou N, Pilott S, et al. Co-activation of STAT3 and YES-associated protein 1 (YAP1) pathway in EGFR-mutant NSCLC. J Natl Cancer Inst 2017;109. doi: 10.1093/jnci/djx014.

3. Furst DE. Mechanism of action, pharmacology, clinical efficacy and side effects of auranofin. An orally administered organic gold compound for the treatment of rheumatoid arthritis. Pharmacotherapy 1983;3:284-98.

4. Justilien V, Walsh MP, Ali SA, et al. The PRKCI and SOX2 oncogenes are coamplified and cooperate to activate Hedgehog signaling in lung squamous cell carcinoma. Cancer Cell 2014;25:139-51.

Cite this article as: Ito $M$, Codony-Servat $\mathrm{C}$, Karachaliou $\mathrm{N}$, Rosell R. Targeting PKC1-PAK1 in EGFR-mutation positive non-small cell lung cancer. Transl Lung Cancer Res 2019;8(5):667-673. doi: 10.21037/tlcr.2019.08.25
5. Regala RP, Weems C, Jamieson L, et al. Atypical protein kinase $\mathrm{C}$ iota is an oncogene in human non-small cell lung cancer. Cancer Res 2005;65:8905-11.

6. Regala RP, Weems C, Jamieson L, et al. Atypical protein kinase Ciota plays a critical role in human lung cancer cell growth and tumorigenicity. J Biol Chem 2005;280:31109-15.

7. Deacon SW, Beeser A, Fukui JA, et al. An isoformselective, small-molecule inhibitor targets the autoregulatory mechanism of p21-activated kinase. Chem Biol 2008;15:322-31.

8. Wu DW, Wu TC, Chen CY, et al. PAK1 is a novel therapeutic target in tyrosine kinase inhibitor-resistant lung adenocarcinoma activated by the PI3K/AKT signaling regardless of EGFR mutation. Clin Cancer Res 2016;22:5370-82.

9. Jacobsen K, Bertran-Alamillo J, Molina MA, et al. Convergent Akt activation drives acquired EGFR inhibitor resistance in lung cancer. Nat Commun 2017;8:410.

10. Chou TC. Drug combination studies and their synergy quantification using the Chou-Talalay method. Cancer Res 2010;70:440-46.

11. Narayan RS, Fedrigo CA, Brands E, et al. The allosteric AKT inhibitor MK2206 shows a synergistic interaction with chemotherapy and radiotherapy in glioblastoma spheroid cultures. BMC Cancer 2017;17:204.

12. Zhang Z, Lee JC, Lin L, et al. Activation of the AXL kinase causes resistance to EGFR-targeted therapy in lung cancer. Nat Genet 2012;44:852-60.

13. Semenova G, Chernoff J. Targeting PAK1. Biochem Soc Trans 2017;45:79-88.

14. Hou GX, Liu PP, Zhang S, et al. Elimination of stemlike cancer cell side-population by auranofin through modulation of ROS and glycolysis. Cell Death Dis 2018;9:89. 


\section{Supplementary}

Table S1 Antibodies used in the study

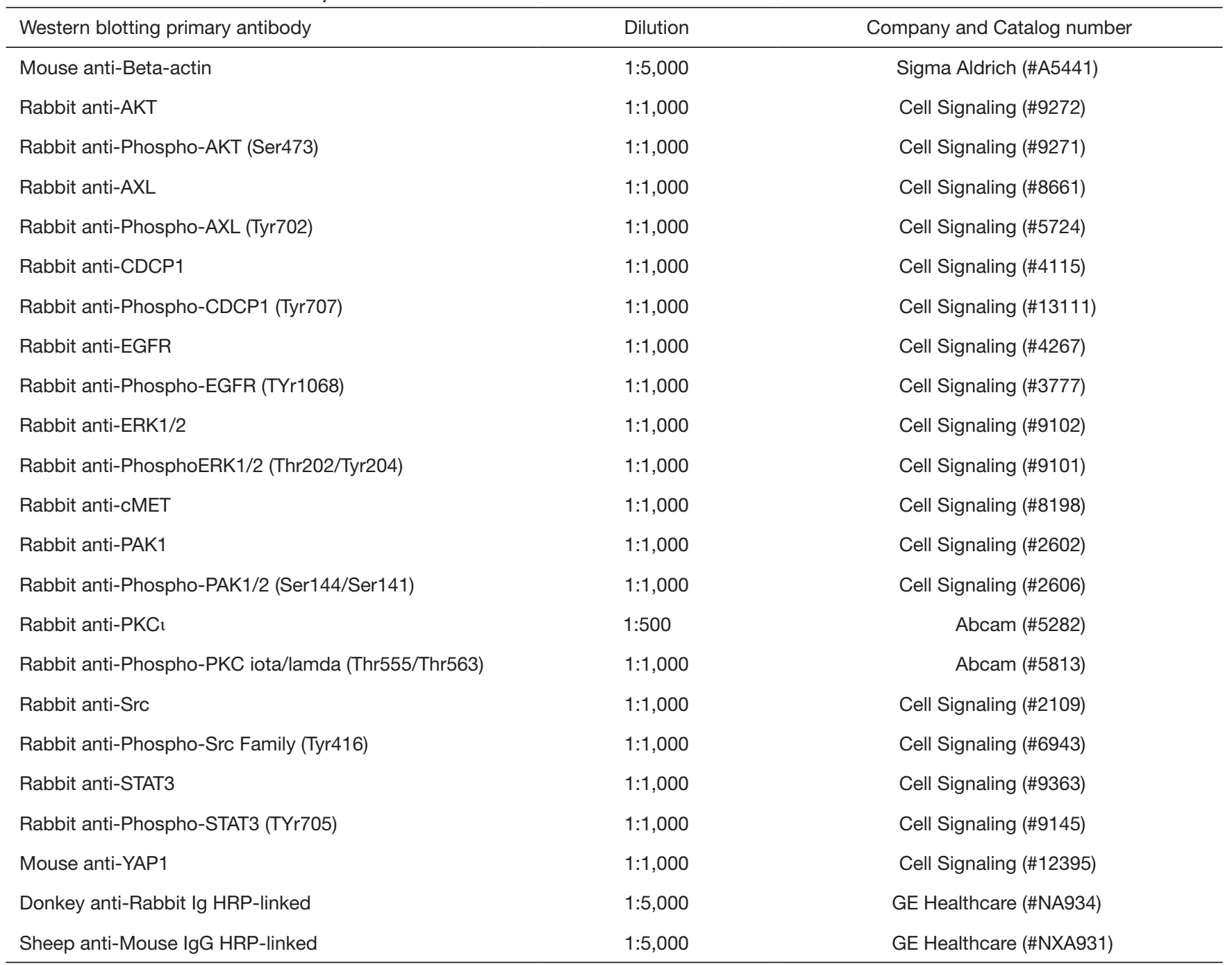

